\title{
Dynamics of droplet formation at T-shaped nozzles with elastic feed lines
}

\author{
Daniéll Malsch · Nils Gleichmann · Mark Kielpinski • \\ Günter Mayer · Thomas Henkel · Dirk Mueller · \\ Volkert van Steijn · Chris R. Kleijn · Michiel T. Kreutzer
}

Received: 18 March 2009/Accepted: 30 June 2009/Published online: 15 August 2009

(c) The Author(s) 2009. This article is published with open access at Springerlink.com

\begin{abstract}
We describe the formation of water in oil droplets, which are commonly used in lab-on-a-chip systems for sample generation and dosing, at microfluidic Tshaped nozzles from elastic feed lines. A narrow nozzle forms a barrier for a liquid-liquid interface, such that pressure can build up behind the nozzle up to a critical pressure. Above this critical pressure, the liquid bursts into the main channel. Build-up of pressure is possible when the fluid before the nozzle is compressible or when the channel that leads to the nozzle is elastic. We explore the value of the critical pressure and the time required to achieve it. We describe the fluid flow of the sudden burst, globally in terms of flow rate into the channel and spatially resolved in terms of flow fields measured using micro-PIV. A total of three different stages - the lag phase, a spill out phase, and a linear growth phase — can be clearly discriminated during droplet formation. The lag time linearly scales with the
\end{abstract}

Electronic supplementary material The online version of this article (doi:10.1007/s10404-009-0479-5) contains supplementary material, which is available to authorized users.

D. Malsch · N. Gleichmann - M. Kielpinski - G. Mayer ·

T. Henkel $(\bowtie)$

Microfluidics Department, Institute of Photonic Technology

(IPHT), Albert Einstein Straße 9, 07745 Jena, Germany

e-mail: thomas.henkel@ipht-jena.de

URL: http://www.ipht-jena.de

\section{Mueller}

Department of Laser Diagnostics,

Institute of Photonic Technology (IPHT),

Albert Einstein Straße 9, 07745 Jena, Germany

V. van Steijn · C. R. Kleijn · M. T. Kreutzer

Faculty of Applied Sciences,

J.M. Burgers Centre for Fluid Mechanics,

Delft University of Technology,

Julianalaan 136, Delft 2628 BL, The Netherlands curvature of the interface inside the nozzle and is inversly proportional to the flow rate of the dispersed phase. A complete overview of the evolution of the growth of droplets and the internal flow structure is provided in the digital supplement.

Keywords Segmented flow - Droplet formation · Micro particle image velocimetry $(\mu \mathrm{PIV}) \cdot$ Micro channel Oil/water two phase flow - Digital reaction technology . Droplet-based microfluidics platform

\section{Introduction}

Microscale-sized droplets and bubbles, generated with precision and transported through microfluidic networks, are ubiquitous in the analysis and synthesis of biomedical and chemical materials (Günther and Jensen 2006; Haeberle and Zengerle 2007; Song et al. 2006; Teh et al. 2008). The most common situation is a stream of liquid carrying immiscible droplets or bubbles that have a cross-sectional area comparable to that of the channels. The fluid compartments in such a segmented flow (Henkel et al. 2004; Kreutzer et al. 2005) behave as individual vessels, each of them containing a different sample or experimental condition. In our work, we use channels with a nearly circular cross section. Droplets in these channels fully conform to the walls as opposed to channels with a rectangular cross section commonly used in this field. As a result, the connectivity and exchange between compartments containing the continuous phase are much less in our channels compared to channels with a rectangular cross section (Kreutzer et al. 2008).

Key to the success of microfluidic applications based on segmented flow is the control of fluid compartments on an individual level. Motivated by this necessity, a field of 
research emerged that explores and develops methods to reproducibly generate (Garstecki et al. 2006; Guillot and Colin 2005; Henkel et al. 2004; Malsch et al. 2008a, b; Nisisako et al. 2002; Song et al. 2003; Thorsen et al. 2001; Zheng et al. 2004), fuse (Gleichmann et al. 2008; Kielpinski et al. 2006; Tan et al. 2004) and split droplets (Kielpinski et al. 2008; Link et al. 2004; Pollack et al. 2002), dose liquid into droplets (Henkel et al. 2004), sort and direct them (Cristobal et al. 2006; Engl et al. 2005; Schindler and Ajdari 2008) and perform phase separation (Kralj et al. 2007; Voigt et al. 2007). In addition, "logic" operations were developed (Prakash and Gershenfeld 2007), which have been successfully applied in smart operation units that implement fluidic closed-loop self-control for autonomous operation (Kielpinski et al. 2008). Integration of such operations opens a way to design entire laboratories on chips based on segmented flow (Gleichmann et al. 2008).

In this work, we consider the formation of droplets at Tjunctions under circumstances typical to multiphase microfluidics (Christopher and Anna 2007): interfacial forces dominating over viscous, inertial and gravitational forces, and fluid streams confined by the geometry of the junction. In this regime, often referred to as dripping or squeezing (Garstecki et al. 2006), a droplet is created as follows: the tobe-dispersed phase-injected from the side branch of the junction-enters the main channel, conforms to the geometry of the junction and gets pushed downstream by the continuous phase until pinch-off occurs. Herein, we mainly focus on the creation of droplets having the smallest possible volume required to occupy the cross section of the main channel. We refer to this volume as the 'Ideal Minimum Compartment Volume' (IMCV). In previous work (Henkel et al. 2004), we demonstrated that this targeted droplet size can be achieved in T-junctions with an integrating nozzle.

The question we address in this article is the following: Can we control the dynamics during the initial stage of droplet growth in which the nascent droplet enters the main channel, while feeding the fluids at fixed rates-of-flow? The answer to this question has direct implications for the improvement of the efficiency of micro mixing inside forming droplets. We show that the speed with which the dispersed phase spills into the main channel can be controlled by the geometry of the nozzle integrated in our Tjunctions in case we include a flexible feed line in our channel network. We provide a detailed analysis of the dynamics of droplet formation for four nozzle geometries and several flow conditions. We used two complementary experimental techniques. High-speed imaging was used to describe the evolution globally in terms of the flow rate through the nozzle, and micro particle image velocimetry ( $\mu$ PIV) (Santiago et al. 1998) was used to measure the evolution of the flow patterns inside growing droplets. The $\mu$ PIV technique was previously used to measure the evolution of the flow around bubbles created at T-junctions (van Steijn et al. 2007) as well as to characterize mixing inside steadily moving droplets transported through straight and meandering channels (Günther et al. 2005; Kinoshita et al. 2007; Sarrazin et al. 2006).

\section{Experimental}

\subsection{Design of microfluidic chip}

We designed a network of micro channels that consists of one main channel connected to four side channels through T-shaped nozzles as shown in Fig. 1a. The main channel has an almost round cross section with thin coplanar faces as depicted in Fig. $1 \mathrm{~b}$ and c. The width $w$ and height $h$ are $300 \mu \mathrm{m}$ and $260 \mu \mathrm{m}$, respectively. The side channels are connected to the main channel through nozzles with an almost round cross section. The dimensions of these nozzles (width $w_{\mathrm{n}}$ and height $h_{\mathrm{n}}$ ) are listed in Table 1 .

A constructional parameter to characterize the size of droplets flowing through micro channels is the minimum volume required to occupy the cross section of the main channel $V_{\mathrm{IMCV}}$. Droplets with a volume larger than $V_{\mathrm{IMCV}}$ are transported through the channel in plug flow. The compartments containing the continuous fluid are only interconnected through the thin lubricating film along the walls of the channel, and exchange between droplets is absent. The volume $V_{\mathrm{IMCV}}$ is calculated from the body of revolution of the channel cross section $V_{\mathrm{IMCV}}=2 \pi \int_{z=0}^{h / 2} r(z)^{2} \mathrm{~d} z$, with $r(z)$ given in Fig. 1c. In this work, the volume of droplets created at our nozzles is somewhat smaller than $V_{\mathrm{IMCV}}=13 \mathrm{~nL}$.

\subsection{Fabrication of all-glass microfluidic device}

The device has been prepared with our technology of wet etching and anodic bonding of two glass substrates using a bond support layer (Henkel et al. 2004). In brief, we etched channels in two glass substrates. Optionally, a two-step etching process can be used to create channels with different etch depths on a single substrate. We constructed closed channels by bonding the two glass substrates using an anodic bonding process mediated by a silicon bond support layer. This method allows the fabrication of optically transparent devices suitable for image-based analysis of fluid dynamics and process diagnostics, with channel depths up to $320 \mu \mathrm{m}$.

After fabrication, micro channel surfaces are functionalized to achieve optimum wettability for the carrier fluid and minimum wettability for the aqueous droplets. After this treatment, a permanent lubrication layer of oil prevents direct contact between the aqueous droplets and the channel walls. We functionalized the channels as follows: first, 
Fig. 1 a Top-view of the allglass microfluidic device. Oil is injected into the main channel (width $w$, height $h$ ) at a fixed rate of flow $q_{\mathrm{c}}$. An immiscible aqueous solution is injected in one of the side channels (the others being closed) at a rate $q_{\mathrm{d}}$. b SEM image of a T-shaped nozzle (width: $w_{\mathrm{n}}$, height: $h_{\mathrm{n}}$ ) that connects the side channel to the main channel. Channels and nozzles have an almost round cross section as displayed in $\mathbf{c}$ and $\mathbf{d}$. The smallest volume of a droplet that seals the main channel $V_{\mathrm{IMCV}}$ is calculated from the body of revolution of the cross section of the main channel as displayed in c

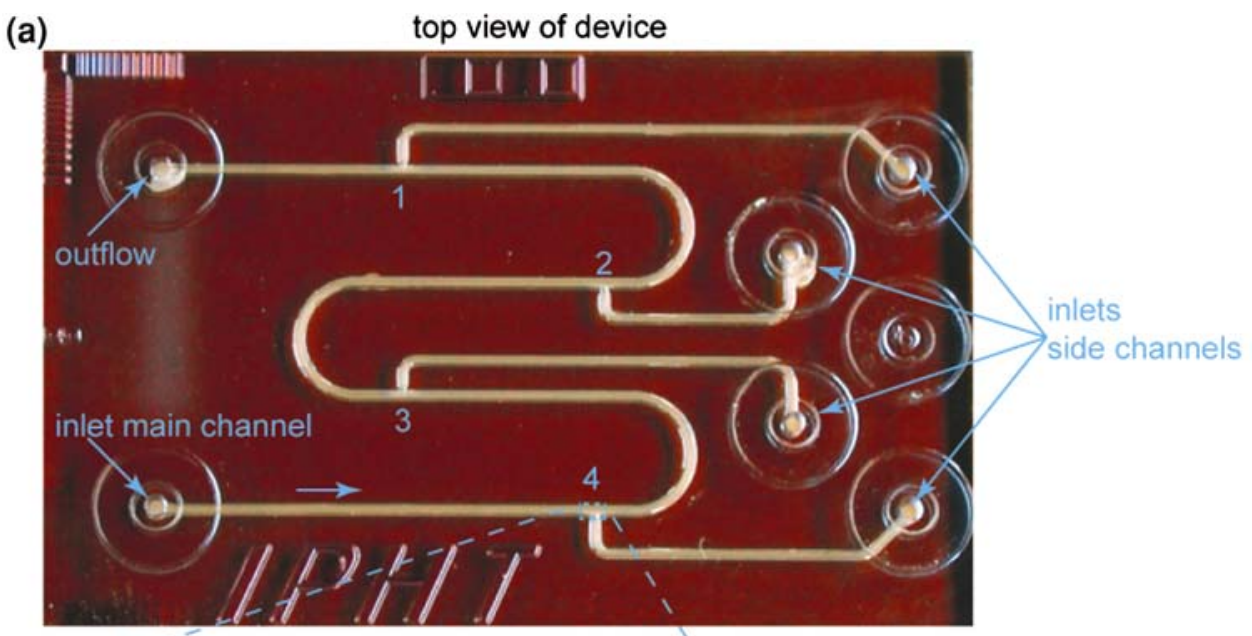

(c) axis of revolution
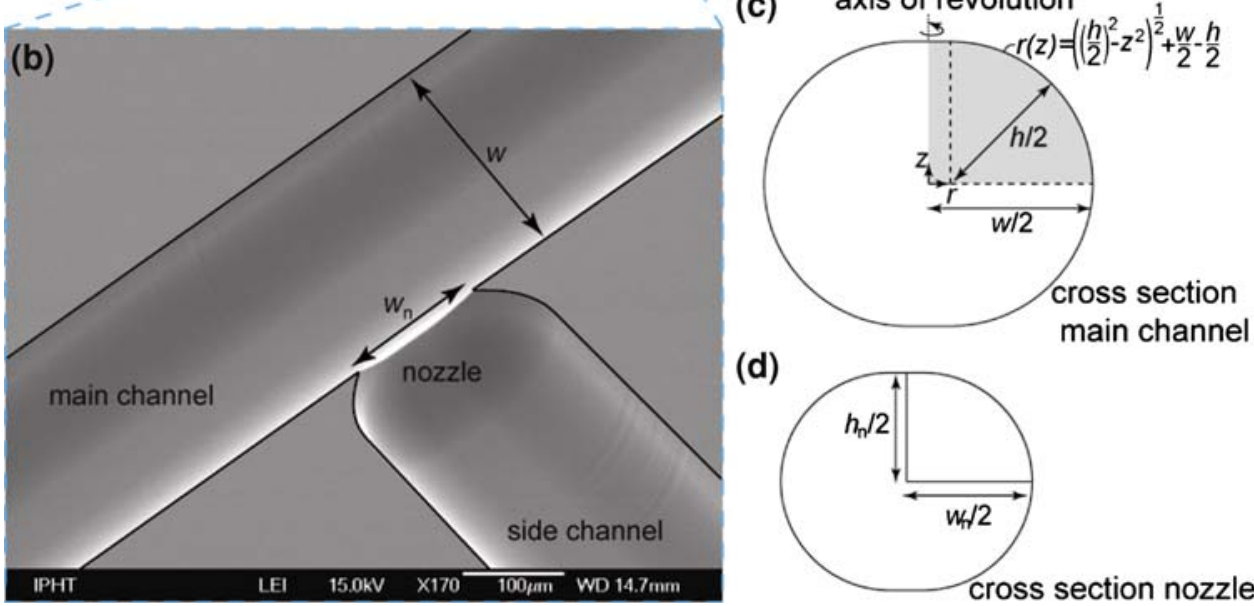

Table 1 Dimensions of the nozzles connecting the four side channels to the main channel

\begin{tabular}{llll}
\hline Nozzle & $w_{\mathrm{n}}(\mu \mathrm{m})$ & $h_{\mathrm{n}}(\mu \mathrm{m})$ & $\kappa\left(10^{4} \mathrm{~m}^{-1}\right)$ \\
\hline 1 & 126 & 86 & 8.7 \\
2 & 104 & 72 & 6.7 \\
3 & 74 & 50 & 4.7 \\
4 & 58 & 38 & 3.9 \\
\hline
\end{tabular}

The dimensions of the main channel are $300 \times 260 \mu \mathrm{m}^{2}$. Nozzle cross sections range from 2.7 to $13.4 \%$ of the main channel cross section. The curvature $\kappa$ of a liquid-liquid interface at a nozzle is imposed by the nozzle geometry and calculated as $\kappa=2 / w_{\mathrm{n}}+2 / h_{\mathrm{n}}$

we removed the support layer used to bond the substrates by exposing the channels for $5 \mathrm{~min}$ to a solution of $5 \mathrm{~mol} / \mathrm{L}$ $\mathrm{NaOH}$ in water at $80^{\circ} \mathrm{C}$, followed by washing steps with a solution of a $0.1 \mathrm{~mol} / \mathrm{L} \mathrm{NaOH}$ and water. Second, the channel walls were activated by exposing them to piranha solution for $10 \mathrm{~min}$ and, subsequently, dried at $120^{\circ} \mathrm{C}$. Finally, surface functionalization was done by exposing the walls for $4 \mathrm{~h}$ to a solution of $5 \mathrm{mmol} / \mathrm{L}$ octadecyl-trichlorosilane (ODTS) in anhydrous tetradecane at $60^{\circ} \mathrm{C}$, followed by washing steps with n-heptane, methanol, and water. The same treatment was used to clean and refunctionalize the channels.

\subsection{Experimental procedure and conditions}

We injected the carrier stream into the main channel at a rate of flow $q_{\mathrm{c}}$, while the to-be-dispersed fluid was fed into one of the side channels at a rate $q_{\mathrm{d}}$. Before the start of an experiment, we filled all side channels, except the one of interest, with carrier fluid and carefully closed their inlets by introducing needles into the Teflon supply lines, thereby making sure no air was left behind. The difference in pressure drop over the main channel is relatively small compared to the pressure required to push an aqueous droplet through a nozzle, such that a comparison between the dynamics of droplet growth at different nozzles, although located at different positions on the chip, is possible.

Fluid dosing was done using a ceDOSYS four channel high precision syringe pump (Cetoni $\mathrm{GmbH}$, Korbußen) equipped with $2.5 \mathrm{~mL}$ syringes (ILS Germany, Stützerbach). The fluidic connections were realized with HPLC 
Teflon capillaries $\left(1 / 16^{\prime \prime}\right.$ outer diameter, $0.50 \mathrm{~mm}$ inner diameter).

We performed experiments with a fixed ratio between the flow rate of the continuous and dispersed phase $q_{\mathrm{c}}$ l $q_{\mathrm{d}}=5$. We varied the total flow rate $q_{\mathrm{c}}+q_{\mathrm{d}}$ between 0.3 and $2.4 \mu \mathrm{L} / \mathrm{s}$. For these operating conditions, micro droplets have a volume of $V=6-12 \mathrm{~nL}$ and are produced at rates ranging from 4 to $60 \mathrm{~s}^{-1}$.

Characteristic dimensionless numbers describing the relative importance of interfacial, viscous, inertial, and gravitational forces are the Capillary (Ca), Weber (We), Reynolds (Re), and Bond (Bo) number. The values of these numbers, based on a total flow rate of $1.2 \mu \mathrm{L} / \mathrm{s}$ and the fluid properties of the dispersed phase given in the next section, are listed in Table 2. These values show that interfacial forces dominate viscous, inertial, and gravitational forces.

In a separate experiment, we measured the pressure required to push a static liquid-liquid interface through a nozzle for each nozzle in our device. The interface is pushed through the nozzle when the hydrostatic pressure at the nozzle balances the Laplace pressure

$\rho g H=\gamma \kappa$,

with $\rho$ the density, $g$ the gravitational acceleration, $H$ the height of the liquid, $\gamma$ the interfacial tension, and $\kappa$ the nozzle curvature listed in Table 1. A comparison between the measured hydrostatic pressure and calculated Laplace pressure is shown in the parity plot in Fig. 2. This plot shows that the pressure required to push a static interface with the curvature set by the geometry of the nozzle equals the Laplace pressure.

\subsection{Refractive index matching}

It is essential in our work to match the indices of refraction of the fluids and material of the channel walls to be able to accurately resolve the flow inside the droplet close to the liquid-liquid interface and close to the channel walls (Budwig 1994). The refractive index of our glass devices is $n=1.470$ (at the wavelength of illumination $\lambda=625 \mathrm{~nm}$ ) according to the data sheet (BOROFLOAT ${ }^{\circledR} 33$ borosilicate glass, Schott AG). This sets the targeted index of refraction for our fluids.

The working fluids are decahydronaphthalene (cis + trans decalin $>98 \%$ from Fluka, $n=1.474$ ) for the continuous phase and a solution of $54.6 \mathrm{wt} \%$ ammonium

Table 2 Typical values of the Capillary, Weber, Bond, and Reynolds number for our experiments

\begin{tabular}{llll}
\hline $\mathrm{Ca}$ & We & Bo & Re \\
\hline $8 \times 10^{-4}$ & $2 \times 10^{-3}$ & $2 \times 10^{-2}$ & 2.8 \\
\hline
\end{tabular}

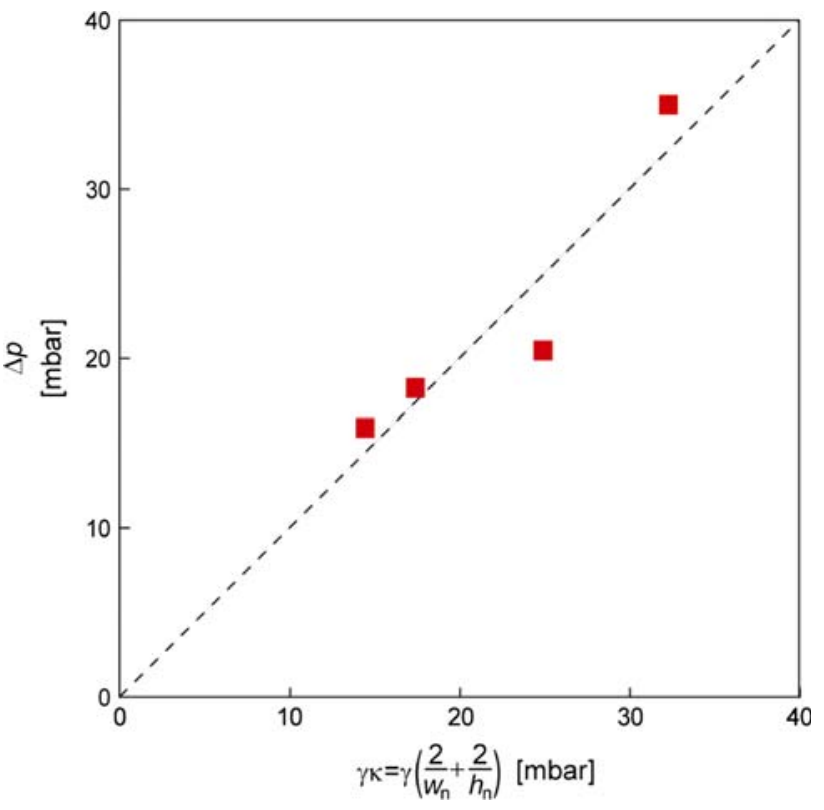

Fig. 2 Parity plot showing a comparison between the measured hydrostatic pressure and the calculated Laplace pressure required to push a static liquid-liquid interface through. The four data points correspond to the four nozzles. We calculated the Laplace pressure using the interfacial tension and curvature at the nozzle listed in Table 1

thiocyanate (NH4SCN $>99 \%$, Bernd Kraft $\mathrm{GmbH}$ ) in deionized water for the dispersed phase. In order to match the indices of refraction, we added a low amount of tetradecane $(n=1.43)$ to the continuous phase, whereas $4.5 \mathrm{wt} \%$ sodium hydroxide was added to the dispersed phase. Both mixtures have an index of refraction $n=1.470$, which was measured using a digital Abbe refractometer. The viscosity of both mixtures is relatively low. Details on fluid properties are listed in Table 3.

\section{$2.5 \mu \mathrm{PIV}$ experiments and flow characterization}

We characterized the flow inside the forming droplets using micro particle image velocimetry. The dispersed

Table 3 Properties of the fluid mixtures

\begin{tabular}{llllll}
\hline Liquid & $n$ & $\rho\left[\mathrm{g} / \mathrm{cm}^{3}\right]$ & $\mu[\mathrm{mPas}]$ & $\sigma[\mathrm{mN} / \mathrm{m}]$ & $\gamma[\mathrm{mN} / \mathrm{m}]$ \\
\hline Decalin & 1.47 & 0.88 & 2.5 & 30 & 38 \\
$\mathrm{NH}_{4} \mathrm{SCN}$ solution & 1.47 & 1.16 & 1.8 & 37 &
\end{tabular}

The surface tension $\sigma$ of the $\mathrm{NH}_{4} \mathrm{SCN}$ solution and the liquid-liquid interfacial tension $\gamma$ were measured using the bubble pressure method with an orifice with a diameter of $0.32 \mathrm{~mm}$. The viscosity $\mu$ was measured using a capillary viscosimeter, using a capillary with a diameter of $0.25 \mathrm{~mm}$ and a length of $1.2 \mathrm{~m}$. Density $\rho$ was measured by gravimetry. Refractive indices were measured using an Abbe refractometer. The surface tension of decalin was taken from Jasper (1972) 
phase was seeded with polystyrene micro particles having a diameter of $2 \mu \mathrm{m}$ (BS-Partikel GmbH, Germany). These particles, delivered in an aqueous solution containing tensides, were added to our refractive index-matched solution after suitable washing steps. The particle concentration was $1 \times 10^{9}$ particles per $\mathrm{mL}$ yielding $\sim 10$ distinguishable particles inside an interrogation window. We visualized the flow in dark field transmission mode using a custom-build optical setup, comprising a microscope lens (magnification $M=20 \times$, numerical aperture NA $=0.4$ ) and a C-mount adapter $(M=2 \times)$ resulting in an overall magnification of $40 \times$. The depth of focus $\delta z$ of our system can be calculated as (Inoue and Spring 1997)

$\delta z=\frac{n \lambda_{0}}{\mathrm{NA}^{2}}+\frac{n \cdot e}{\mathrm{NA} \cdot M}$,

with $n$ the refractive index of air, $\lambda_{0}$ the wavelength of illumination in vacuum, NA the numerical aperture, $e$ the pixel spacing of the CCD sensor, and $M$ the total magnification. For our setup, the depth of focus is $\delta z=5 \mu \mathrm{m}$. In all our $\mu$ PIV measurements, we centered the focal plane of the microscope lens at the midplane of our micro channel by focusing on the edge of the silicon bond support layer that is visible in the images and located exactly at the plane-of-symmetry of the channel. The spatial resolution in the out-of-plane direction is estimated from the depth of correlation $\delta z_{\text {corr }}$ (Wereley and Meinhart 2005) as $\delta z_{\text {corr }}=10 \mu \mathrm{m}$.

We acquired $\mu \mathrm{PIV}$ images with a high-speed camera (Photron Fastcam Ultima APX $\mathrm{I}^{2} 120 \mathrm{~K}$ ) at a rate of 2000 frames per s with an image resolution of $1024 \times$ $1024 \mathrm{px}^{2}$. These images were evaluated with software developed in cooperation with the Department of Computer Science at Friedrich-Schiller-University, Jena. The employed algorithm is based on direct cross correlation guided by the shift detection by restoration method. The location of the correlation peak was determined using a Gaussian interpolation scheme with a sub-pixel resolution of $\sim 0.1 \mathrm{px}$. The time between subsequent images was set such that the minimum displacement of particles is at least $5 \mathrm{px}$ resulting in a relative uncertainty in the displacement field of $2 \%$. The ratio between the largest peak and second largest peak in a correlation plane, often referred to as peak ratio or Q-ratio, is a measure for the quality of the acquired and evaluated PIV images and typically ranged between 1.5 and 1.8 in our measurements. The final size of the interrogation windows is $32 \times 32 \mathrm{px}^{2}$, resulting in an in-plane spatial resolution of $14 \times 14 \mu^{2}$ for the lower flow rate, whereas for the higher flow rates, we used a window of $64 \times 64 \mathrm{px}^{2}$. We applied a 50\% overlap among the interrogation windows.

We post-processed the velocity fields using a median filter similar to the one described by Westerweel and Scarano 2005. The algorithm selects the correlation peaks based on the notion that the velocity field is smooth. We replaced vectors having a peak ratio below 1.2 using this median filter. Typically, less than $5 \%$ of the vectors were corrected in this post-processing step. Further details of the setup and employed algorithms have been published previously (Malsch et al. 2008a, b).

We recorded the evolution of the droplet volume using bright field microscopy in transmission mode. We used the same camera and recorded the evolution at 4000 frame$\mathrm{s}$ per $\mathrm{s}$ with a resolution of $1024 \times 512 \mathrm{px}^{2}$. We derived the three-dimensional volume of a droplet from twodimensional pictures as follows. According to the Lambert-Beer law, the fraction of light absorbed in a liquid linearly depends on the path length as

$A=\varepsilon l c$,

with $A$ the absorbance, $\varepsilon$ the extinction coefficient, $l$ the path length, and $c$ the concentration of the compound in solution. In order to enhance absorption, we added a dye (bromophenol blue) to the dispersed phase. For our refractive index-matched system in which refraction at interfaces is absent, the gray levels in our images contain information on the third dimension of a droplet. We calculated the droplet volume from the background-corrected image using the gray value integral over the droplet area and a proportionality factor $\alpha$. This proportionality factor $\alpha$ is based on the linear correlation between the optical path length $l$ and the absorbance $A$. It is calculated from the gray value intensity $I_{\max }$ at the position of the maximum micro channel height as $\alpha=h_{\max } / I_{\max }$.

\section{Results}

\subsection{Dynamics of droplets growing at T-shaped nozzles}

In this section, we show how droplets are formed at T-shaped nozzles in case the dispersed phase is supplied to the micro channel through an elastic feed line. We present the evolution of the volume $V$ of a forming droplet in Fig. 3a. The volume was derived from gray values in our high-speed images as discussed in the previous section. The vertical axis on the right side shows these values in arbitrary units.

The formation of a droplet is characterized by three distinct periods, $t_{\mathrm{lag}}, t_{\text {burst }}$, and $t_{\text {linear. }}$. During the first period $t_{\text {lag }}$, the dispersed phase does not leave the nozzle although it is being supplied at a constant rate $q_{\mathrm{d}}$. At $t=57 \mathrm{~ms}$, the dispersed phase abruptly bursts into the main channel. The volume $V$ increases at a rate larger than $q_{\mathrm{d}}$ during a period $t_{\text {burst }}$. At $t=76 \mathrm{~ms}$, the volume increases linearly with time at a rate $\mathrm{d} V / \mathrm{d} t \sim q_{\mathrm{d}}$ until the droplet eventually pinches-off. In contrast to the creation of droplets in 


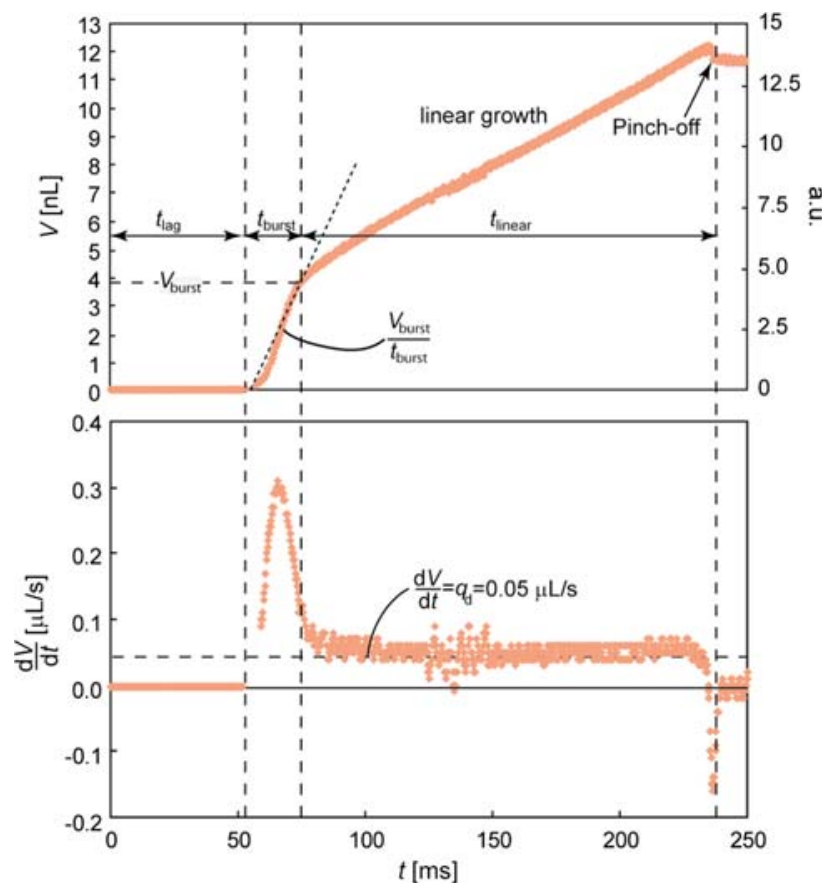

Fig. 3 Top Volume of a forming droplet as a function of time. The formation is characterized by three distinct period, $t_{\text {lag }}, t_{\text {burst }}$, and $t_{\text {linear }}$. The dispersed phase, although supplied at a constant rate $q_{\mathrm{d}}$, does not leave the nozzle during $t_{\text {lag. }}$. At $t=57 \mathrm{~ms}$, the dispersed phase abruptly bursts into the main channel at a rate larger than $q_{\mathrm{d}}$. At $t=76 \mathrm{~ms}$, the volume of the droplet linearly increases at a rate $q_{\mathrm{d}}$ until pinch-off occurs. The values on the vertical axis on the right side represent the gray values (in arbitrary units) measured in our experiments that were used to derive the volume. Bottom The rate $\mathrm{d} V / \mathrm{d} t$ as a function of time. Conditions: $q_{\mathrm{d}}=0.05 \mu \mathrm{L} / \mathrm{s}, q_{\mathrm{c}}=0.25 \mu \mathrm{L} / \mathrm{s}$, nozzle $1\left(w_{\mathrm{n}} \times h_{\mathrm{n}}=\right.$ $126 \times 86 \mu \mathrm{m}^{2}$ )

microfluidic networks that lack elasticity such that fluid streams enter the main channel at constant rates, relatively large difference in the flow rate through the nozzle $\mathrm{d} V / \mathrm{d} t$ was observed in our experiments as shown in Fig. $3 b$.

We now show how the dynamics of the fluid system during the period $t_{\text {lag }}$ depends on the flow rate of the dispersed phase and the geometry of the nozzle. Our hypothesis hereby is that pressure builds-up in the elastic feed line until the pressure at the nozzle becomes larger than the pressure required to push the interface through the nozzle. For an elastic feed line, the pressure inside the line $p_{\text {feed }}$ linearly scales with its expansion and hence with the volume of fluid it contains. In our system, this volume is supplied at a rate $q_{\mathrm{d}}$, and hence build-up of pressure in the feed line progresses as $\mathrm{d} p_{\text {feed }} / \mathrm{d} t \propto q_{\mathrm{d}}$. The pressure $p_{\text {burst }}$ required to push the interface through the nozzle is-as we will address shortly_independent of $q_{\mathrm{d}}$. Hence, this simple scaling analysis shows that the time required to build-up enough pressure in the feed line to push the interface through the nozzle is inversely proportional to $q_{\mathrm{d}}$. Indeed, this relation is observed in our measurements for all nozzles. Figure 4 shows that the time $t_{\mathrm{lag}}$ is inversely proportional to the rate $q_{\mathrm{d}}$ and strongly depends on the geometry of the nozzle. Next, we explain this geometry dependence.

As discussed in the previous section, the pressure $p_{\text {burst }}$ required to push a static interface through a nozzle is equal to the Laplace pressure at the liquid-liquid interface at the nozzle given by $p_{\text {burst }}-p_{\text {ref }}=\kappa \gamma$, with $p_{\text {ref }}$ a reference pressure at the nozzle. The curvature $\kappa$ of the interface is imposed by the nozzle geometry and calculated as $\kappa=$ $\left(2 / w_{n}-2 / h_{n}\right)$. Values of $\kappa$ are listed in Table 1 .

The dispersed phase enters the main channel once $p_{\text {feed }}\left(t=t_{\text {lag }}\right)=p_{\text {burst }}$. With $p_{\text {feed }}\left(t=t_{\text {lag }}\right) \propto q_{\mathrm{d}} t_{\text {lag }}$ and $p_{\text {burst }} \propto \kappa$, our simple scaling analysis predicts that $t_{\text {lag }} \propto \kappa / q_{\mathrm{d}}$.

In Fig. 5 we analysed the length of the lag time period $t_{\text {lag }}$ dependent on maximum curvature of the interface inside the nozzle $\kappa$ and the flow rate of the dispersed phase $q_{\mathrm{d}}$. Therefore, we plotted $t_{\text {lag }}$ as a function of $\kappa / q_{\mathrm{d}}$. The experimental data obtained for different nozzle geometries and flow rates all collapses onto a single straight line. This leads to the conclusion that pressure builds up in the feed line until the pressure exceeds the Laplace pressure at the nozzle.

We now focus on the dynamics of the 'burst' of the droplet into the main channel. We observed enhanced flow through nozzle once the interface is pushed through the nozzle. The rate of flow through the nozzle is-during a period $t_{\mathrm{burst}}$ in which the droplets rapidly grows to a size

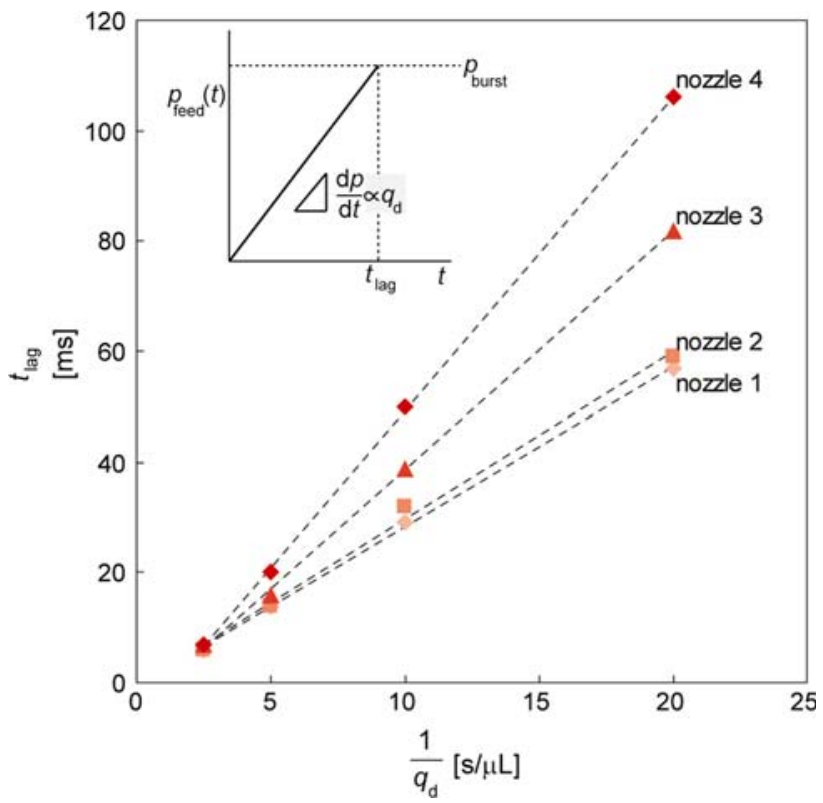

Fig. 4 The period $t_{\text {lag }}$ measured from graphs similar to Fig. 3 is inverse proportional to the flow rate $q_{\mathrm{d}}$ for a given nozzle geometry. For given $q_{\mathrm{d}}$, the period increases with decreasing nozzle size. The dashed linear lines are guides to the eye. The inset shows a sketch of the evolution of pressure inside the feed line. The pressure builds up until the pressure required to push the interface through the nozzle $p_{\text {burst }}$ is reached at $t=t_{\text {lag }}$ 


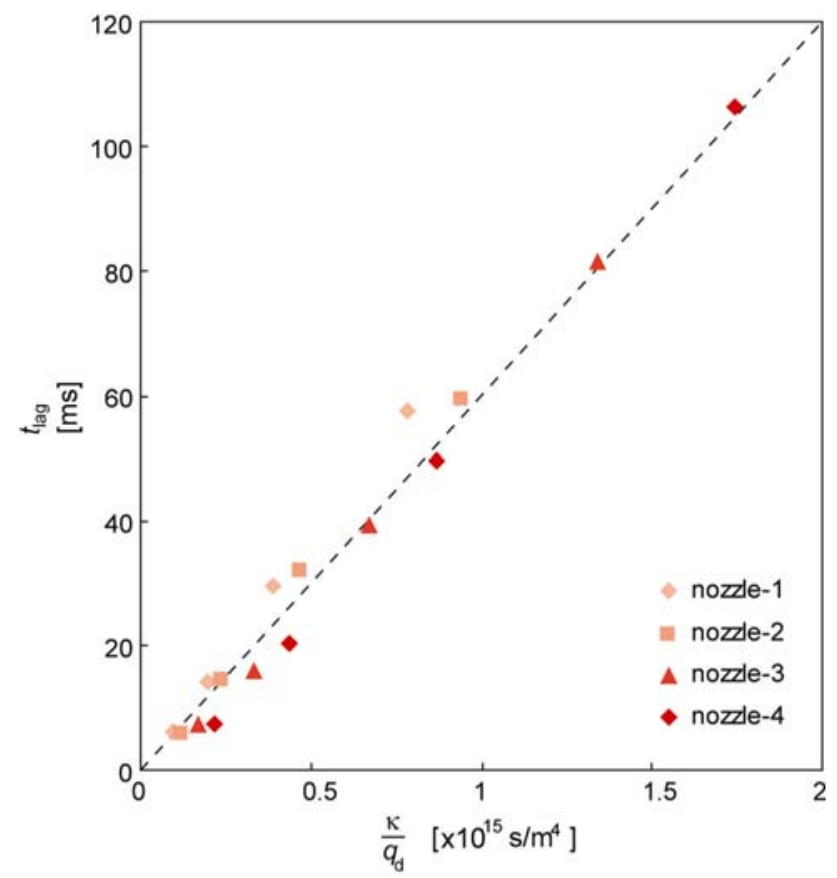

Fig. 5 The period $t_{\text {lag }}$ linearly scales with the curvature $\kappa$ of the interface at the nozzle and is inverse proportional to the flow rate of the dispersed phase $q_{\mathrm{d}}$

$V_{\text {burst }}$ - considerably larger than $q_{\mathrm{d}}$ as shown in Fig. 3. In Fig. 6, we compare the average rate $V_{\text {burst }} / t_{\text {burst }}$ for the four nozzles and several flow rates.

Figure 6 shows that the average normalized rate $V_{\text {burst }} /$ $t_{\text {burst }} q_{\mathrm{d}}$ is significantly larger than 1 for all our nozzles. The

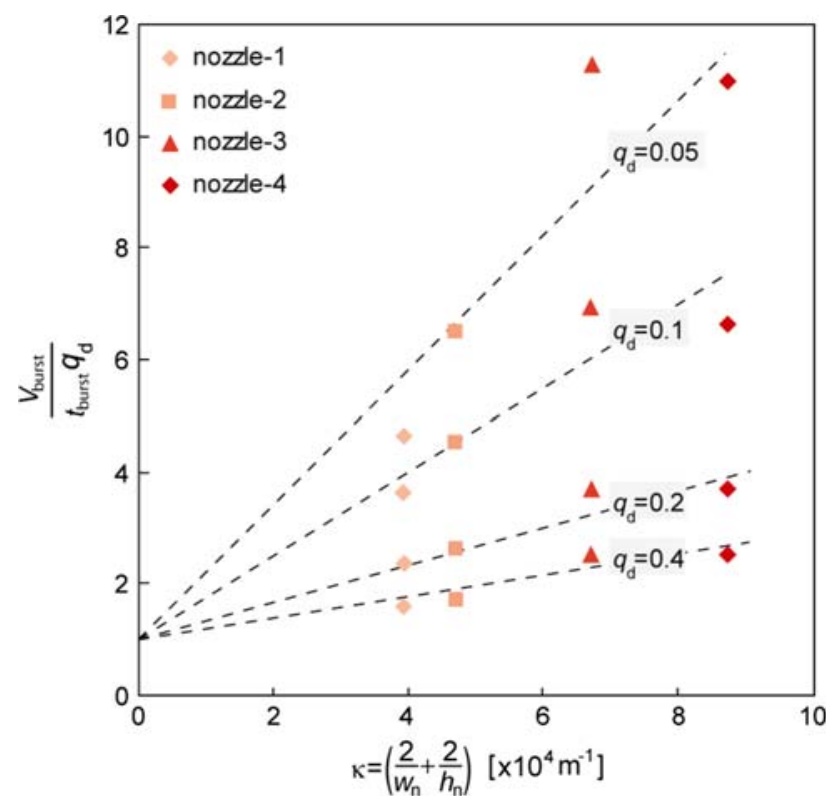

Fig. 6 The average rate $V_{\text {burst }} / t_{\text {burst }}$ at which the forming droplet inflates during $t_{\text {burst }}$, normalized with $q_{\mathrm{d}}$, as a function of the curvature of the nozzle for four flow rates. The dashed lines are guides to the eye that all intersect the vertical axis at $V_{\text {burst }} / t_{\text {burst }} q_{\mathrm{d}}=1$ enhanced flow from the nozzle into the forming droplet depends on both the nozzle geometry and flow rates. Small nozzles hereby lead to relatively large bursts. In our experiments, we observed rates up to $V_{\text {burst }} / t_{\text {burst }} \sim 10 q_{\mathrm{d}}$. The enhanced flow due to build-up and release of pressure in elastic feed lines can be of use in practical applications, where rapid mixing inside forming compartments is key. In the next section, we present the dynamics of the flow inside the forming droplets measured using $\mu$ PIV.

\subsection{PIV measurements of the flow inside droplets}

The evolution of the internal flow inside forming droplets measured using $\mu$ PIV generates additional information, especially on the influence on the nozzle geometry. We performed $\mu \mathrm{PIV}$ measurements on droplets created at nozzles 1 and 3 for a fixed ratio $q_{\mathrm{c}} / q_{\mathrm{d}}=5$ and total flow rates $q_{\mathrm{c}}+q_{\mathrm{d}}=0.3 \mu \mathrm{L} / \mathrm{s}$ and $0.6 \mu \mathrm{L} / \mathrm{s}$. A complete set of PIV videos is available in the electronic supplement. The focal plane was located at the center plane of the channel in all our measurements.

Figure 7 shows a typical raw CCD image with corresponding velocity field. Reconstruction of the velocity field near the entrance of the nozzle is challenging due to the relatively large in-plane and out-of-plane velocities. The obvious way to resolve this issue, without loss of spatial resolution, is to reduce the time between subsequent images. In the current study, this was not possible.

In Fig. 8, we present the velocity distributions inside droplets created at the largest nozzle 1 (left) and the smaller nozzle 3 (right). For both cases, the flow rates are $q_{\mathrm{c}}=0.25 \mu \mathrm{L} / \mathrm{s}$ and $q_{\mathrm{d}}=0.05 \mu \mathrm{L} / \mathrm{s}$. We compare the velocity distributions at four characteristic moments during a formation cycle: halfway the burst period $t=0.5 t_{\text {burst }}$ (Fig. 8a), at the end of the burst period $t=t_{\text {burst }}$ (Fig. 8b), halfway the linear period $t=t_{\text {burst }}+0.5 t_{\text {linear }}$ (Fig. 8c) and prior to pinch-off $t=T$ (Fig. 8d). A first considerable difference between both nozzles is the duration of a full droplet cycle $T$, which is larger for the largest nozzle.

In Fig. 8a, the burst period is clearly recognizable from the velocity distributions. Large velocities, directed in both the upstream and downstream direction, are visible, indicating that the droplet inflates almost symmetrically without being disturbed by the carrier stream of oil. This effect is most pronounced for the smaller nozzle. We observe the largest velocities close to the nozzle; some of the velocity vectors were not resolvable due to the high velocities and out-of-plane movement of particles. In Sect. 3.1, we showed that the flow rate through the nozzle is much larger than $q_{\mathrm{d}}$ during the burst period. Figure 6 shows that the flow rate through nozzle 3 is roughly twice the flow rate through nozzle 1 during the burst period. This velocity difference is clearly visible in our PIV data. 


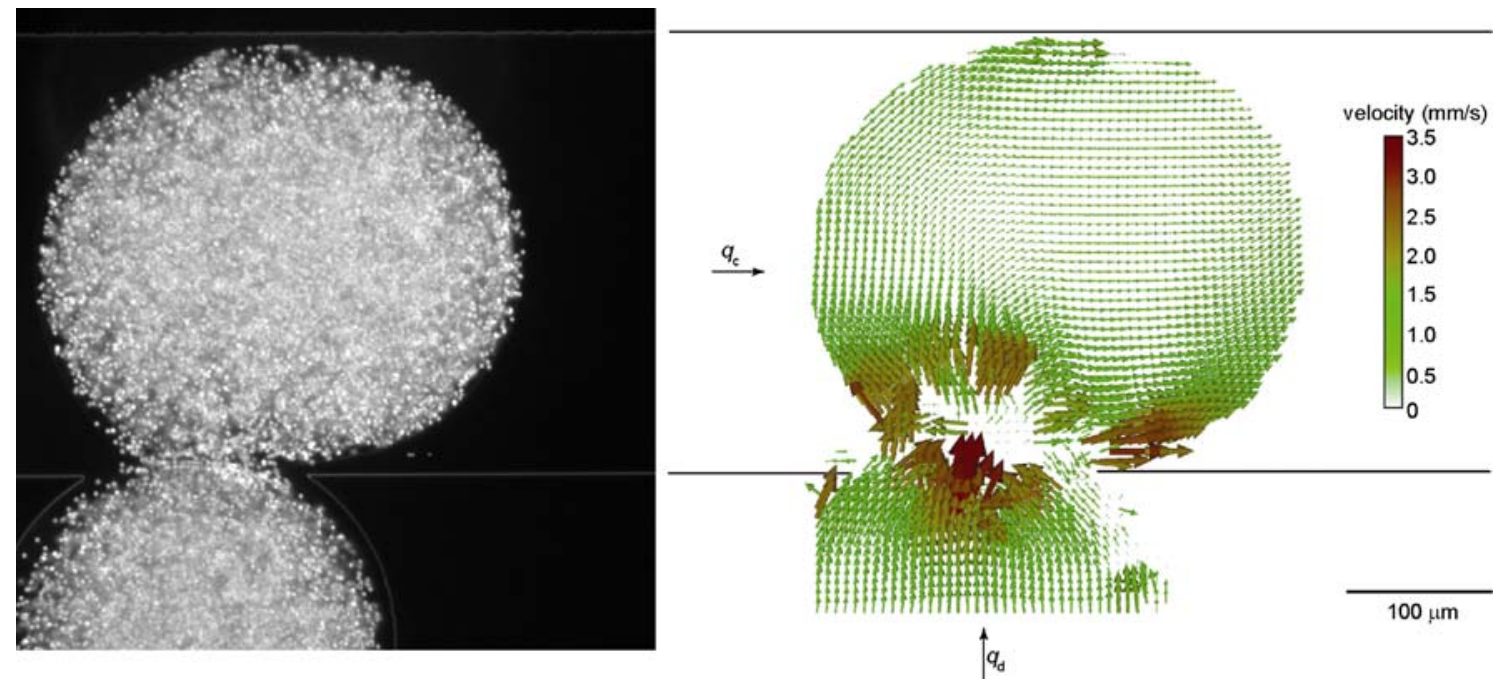

Fig. 7 Left Image taken by the CCD camera. The interface between both fluids and the fluids and walls is invisible, because we matched the indices of refraction. At the channel boundaries, edges of the silicon bond support layer are visible. Right Velocity field

At the end of the burst period (Fig. 8b), we observe the influence of the carrier stream that flows around the droplet and pushes the droplet downstream. The flow is now mainly directed downstream. For the larger nozzle 1, we observe a circular motion inside the droplet, whereas the flow inside the droplet at nozzle 3 is more parallel. Velocities close to the nozzles are still larger than in the linear growth phase that is shown in the next row. Note that the volume of the droplet forming at nozzle 3 is larger compared to the droplet at nozzle 1, although the burst time $t_{\text {burst }}$ is shorter.

Halfway the linear growth phase (Fig. 8c), the droplet formed at the nozzle 1 is still smaller compared to the droplet at nozzle 3. However, the final droplet size is largest for nozzle 1 as can be inferred from Fig. 8d that displays the velocity distribution prior to pinch-off.

After pinch-off, the rapid relaxation of the interfaces induces large velocities resulting in an interesting flow pattern inside the droplet as shown in Fig. 9.

\section{Summary and conclusions}

We described the dynamics of the formation of aqueous droplets at T-shaped nozzles from high-speed camera images and $\mu \mathrm{PIV}$ recordings. We hereby considered the case that the aqueous stream is supplied to our all-glass micro channel through a flexible feed line that allows for build-up and release of pressure. We identified three distinct stages in the formation of a droplet. At the start of a formation cycle, the aqueous phase-although injected into the feed line at a constant rate of flow $q_{\mathrm{d}}$ - does not leave the reconstructed by the PIV algorithm. False velocity vectors near the exit of the nozzle originate from relatively large in-plane and out-ofplane motion of tracer particles

nozzle for a period $t_{\text {lag. }}$. During this period, pressure builds up in the elastically deformable feed line. Based on the hypothesis that the aqueous phase starts flowing through the nozzle once the pressure in the feed line exceeds the pressure at the nozzle, we formulated a simple scaling argument for $t_{\text {lag }}$, which was in excellent agreement with our experimental results. In a second period with duration $t_{\text {burst }}$, the release of pressure causes a relatively large flow through the nozzle inflating the droplet at a rate significantly larger than the supply rate $q_{\mathrm{d}}$. We showed that both the geometry of the nozzle and the flow conditions have a strong influence on the dynamics during this rapid growth. After the burst period, the droplet grows at a rate $q_{\mathrm{d}}$ during a period $t_{\text {linear }}$ until pinch-off occurs. Details on the dynamics of the flow inside the droplets were captured using $\mu \mathrm{PIV}$, where we matched the indices of refraction of the fluids and glass chip to capture the flow near the interfaces.

System elasticity as a tool to control the dynamics of the formation of droplets provides the solution for some common problems in droplet-based microfluidics. For example, in case one wants to fuse a droplet that flows through the main channel with a droplet that forms at a nozzle, mixing of both compartments is enhanced significantly due to the large velocities during the burst period as we observed in unpublished measurements. Another example is the controlled stopping of droplets at strictures and the accelerated release from these strictures. Such applications are, for instance, relevant in droplet-based logic elements or self-operating and self-controlling operation units for lab-on-a-chip applications.

Both examples demonstrate the potential of system elasticity for the development of new operation units for 
nozzle 1

(a)

$$
t=0.5 t_{\text {burst }}
$$

$14 \mathrm{~ms}$

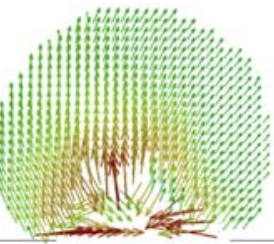

(b)

$$
t=t_{\text {bust }}
$$

$28 \mathrm{~ms}$

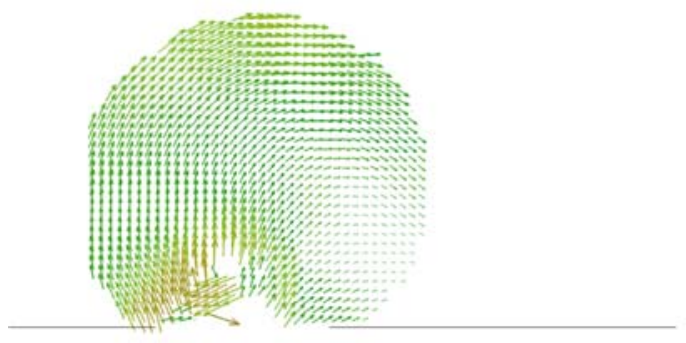

(c)

$$
t=t_{\text {burst }}+0.5 t_{\text {inear }}
$$

$50 \mathrm{~ms}$
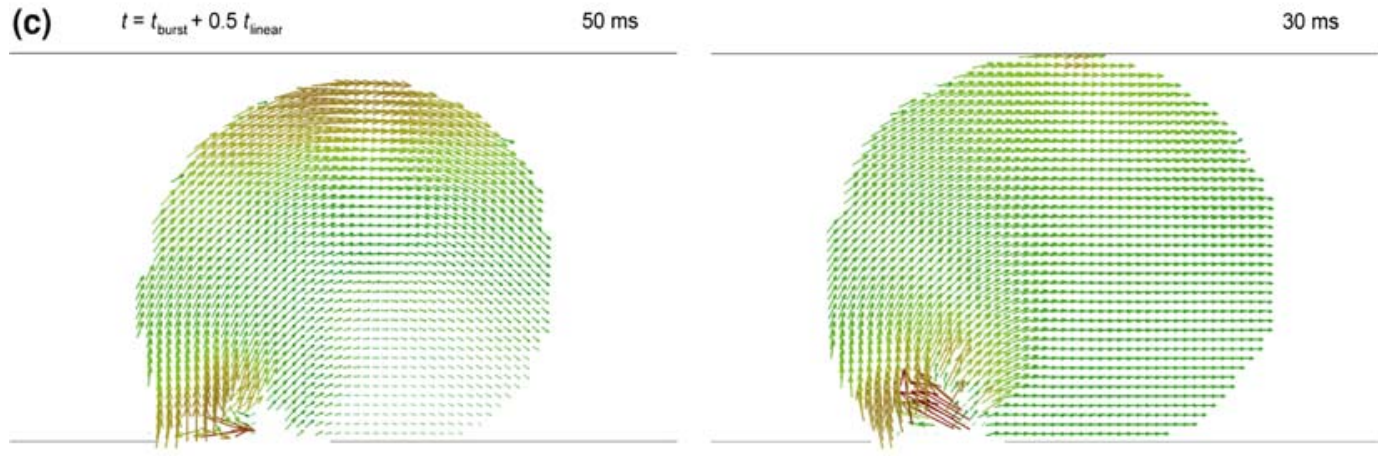

nozzle 3

$9 \mathrm{~ms}$

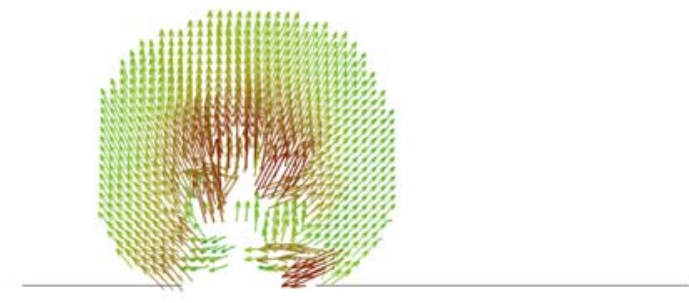

$18 \mathrm{~ms}$

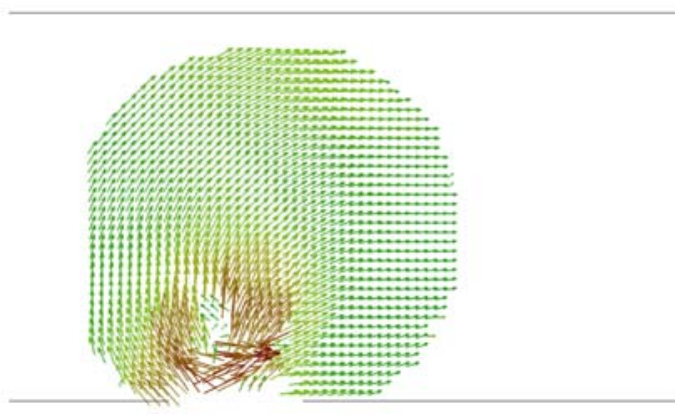

(d)
$t=T$
$73 \mathrm{~ms}$
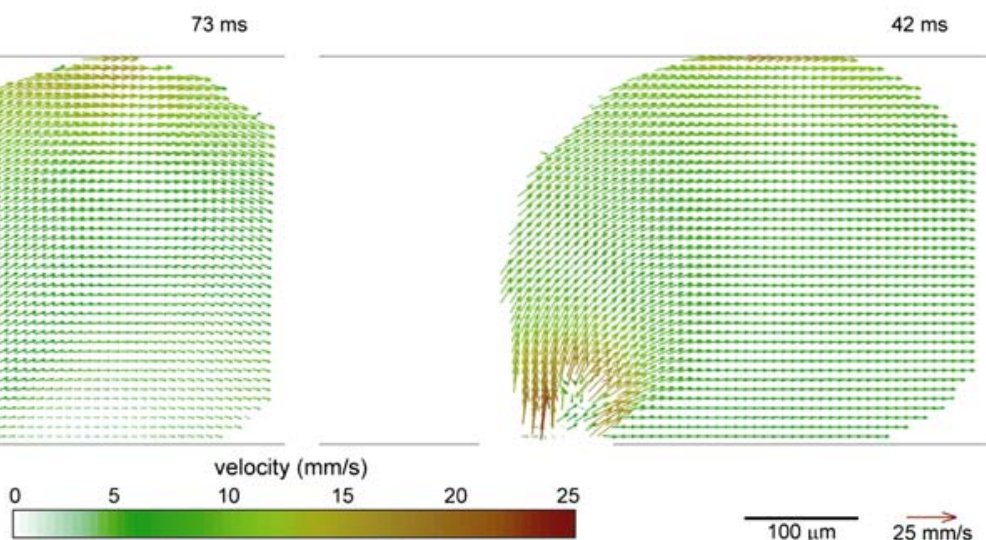

$100 \mu \mathrm{m} \quad 25 \mathrm{~mm} / \mathrm{s}$

Fig. 8 Evolution of the velocity field inside a forming droplet at the larger nozzle 1 (left) and the smaller nozzle 3 (right). Velocity distributions at four characteristic moments during a formation cycle are shown in a-d. Conditions: $q_{\mathrm{d}}=0.05 \mu \mathrm{L} / \mathrm{s}, q_{\mathrm{c}}=0.25 \mu \mathrm{L} / \mathrm{s}$ 


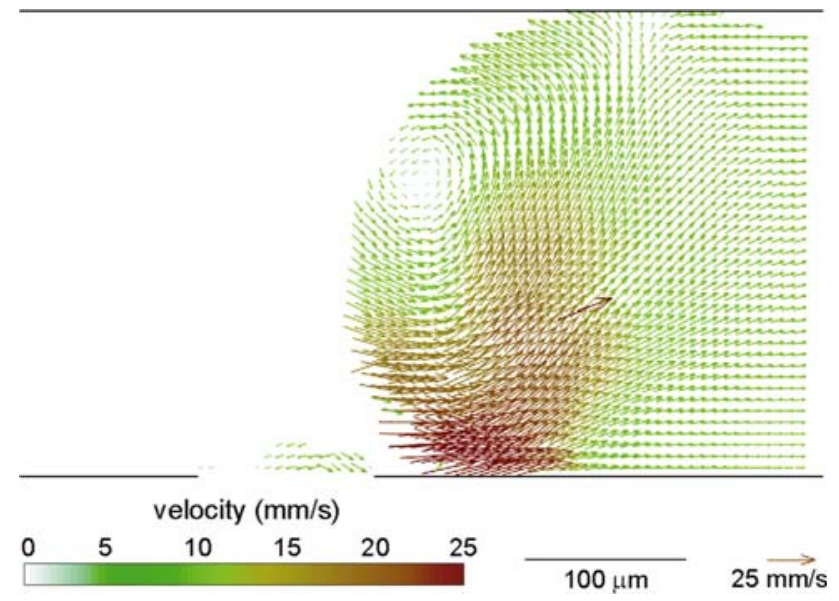

Fig. 9 Velocity distribution inside a droplet created at nozzle 1 shortly after pinch-off. Large velocities are induced by the rapid relaxation of the interfaces resulting in an interesting flow pattern. Conditions: $q_{\mathrm{d}}=0.05 \mu \mathrm{L} / \mathrm{s}, q_{\mathrm{c}}=0.25 \mu \mathrm{L} / \mathrm{s}$

lab-on-a-chip technology and droplet-based logic applications.

Acknowledgments The authors acknowledge the German Bundesministerium für Bildung und Forschung (BMBF) for funding FKZ 16SV1998. The authors also thank the reviewers for their constructive comments that have been considered in enhancements of the experiments and during manuscript revision.

Open Access This article is distributed under the terms of the Creative Commons Attribution Noncommercial License which permits any noncommercial use, distribution, and reproduction in any medium, provided the original author(s) and source are credited.

\section{References}

Budwig RS (1994) Refractive index matching methods for liquid flow investigations. Exp Fluids 17:350-355

Christopher GF, Anna SL (2007) Microfluidic methods for generating continuous droplet streams. J Phys D Appl Phys 40:R319-R336

Cristobal G, Benoit JP, Joanicot M, Ajdari A (2006) Microfluidic bypass for efficient passive regulation of droplet traffic at a junction. Appl Phys Lett 89:034104

Engl W, Roche M, Colin A, Panizza P, Ajdari A (2005) Droplet traffic at a simple junction at low capillary numbers. Phys Rev Lett 95:208304

Garstecki P, Fuerstman MJ, Stone HA, Whitesides GM (2006) Formation of droplets and bubbles in a microfluidic T-junctionscaling and mechanism of break-up. Lab Chip 6:437-446

Gleichmann N, Malsch D, Kielpinski M, Rossak W, Mayer G, Henkel T (2008) Toolkit for computational fluidic simulation and interactive parametrization of segmented flow based fluidic networks. Chem Eng J 135:210-218

Guillot P, Colin A (2005) Stability of parallel flows in a microchannel after a T junction. Phys Rev E 72:066301

Günther A, Jensen KF (2006) Multiphase microfluidics: from flow characteristics to chemical and materials synthesis. Lab Chip 6:1487-1503
Günther A, Jhunjhunwala M, Thalmann M, Schmidt MA, Jensen KF (2005) Micromixing of miscible liquids in segmented gas/liquid flow. Langmuir 21:1547-1555

Haeberle S, Zengerle R (2007) Microfluidic platforms for lab-on-achip applications. Lab Chip 7:1094-1110

Henkel T, Bermig T, Kielpinski M, Grodrian A, Metze J, Köhler J (2004) Chip modules for generation and manipulation of fluid segments for micro serial flow processes. Chem Eng J 101: $439-445$

Inoue S, Spring KR (1997) Video microscopy: the fundamentals, 2nd edn. Plenum Press, New York

Jasper JJ (1972) The surface tension of pure liquid compounds. J Phys Chem Ref Data 1(4):841-948

Kielpinski M, Mayer G, Albert J, Malsch D, Felbel J, Henkel T (2006) Self controlled droplet fusion of segmented sample streams, 9. International Conference on Microreaction Technology, 06.-08.09.2006, Potsdam, Germany, Pub Dechema, p 114

Kielpinski M, Malsch D, Gleichmann N, Mayer G, Henkel T (2008) Application of self-control in droplet-based microfluidics. In: Proceedings of the 6th International Conference on Nanochannels, Microchannels, and Minichannels. ASME, New York, pp 1565-1570

Kinoshita H, Kaneda S, Fujii T, Oshima M (2007) Three-dimensional measurement and visualization of internal flow of a moving droplet using confocal micro-PIV. Lab Chip 7:338-346

Kralj JG, Sahoo HR, Jensen KF (2007) Integrated continuous microfluidic liquid-liquid extraction. Lab Chip 7:256-263

Kreutzer M, Kapteijn F, Moulijn J, Kleijn C, Heiszwolf J (2005) Inertial and interfacial effects on pressure drop of Taylor flow in capillaries. AIChE J 51:2428-2440

Kreutzer MT, Gunther A, Jensen KF (2008) Sample dispersion for segmented flow in microchannels with rectangular cross section. Anal Chem 80:1558-1567

Link DR, Anna SL, Weitz DA, Stone HA (2004) Geometrically mediated breakup of drops in microfluidic devices. Phys Rev Lett 92:054503

Malsch D, Gleichmann N, Kielpinski M, Mayer G, Henkel T (2008a) Effects of fluid and interface interaction on droplet internal flow in all-glass micro channels. In: Proceedings of the 6th International Conference on Nanochannels, Microchannels, and Minichannels. ASME, New York, pp 1571-1578

Malsch D, Kielpinski M, Merthan R, Albert J, Mayer G, Köhler JM, Süße H, Stahl M, Henkel T (2008b) $\mu$-PIV-analysis of Taylor flow in micro channels. Chem Eng J 135:166-172

Nisisako T, Torii T, Higuchi T (2002) Droplet formation in a microchannel network. Lab Chip 2:24-26

Pollack MG, Shenderov AD, Fair RB (2002) Electrowetting-based actuation of droplets for integrated microfluidics. Lab Chip 2: 96-101

Prakash M, Gershenfeld N (2007) Microfluidic bubble logic. Science 315:832-835

Santiago J, Wereley S, Meinhart C, Beebe D, Adrian RJ (1998) A particle image velocimetry system for microfluidics. Exp Fluids 25:316-319

Sarrazin F, Loubiere K, Prat L, Gourdon C, Bonometti T, Magnaudet J (2006) Experimental and numerical study of droplets hydrodynamics in microchannels. AIChE J 52:4061-4070

Schindler M, Ajdari A (2008) Droplet traffic in microfluidic networks: a simple model for understanding and designing. Phys Rev Lett 100:044501

Song H, Tice JD, Ismagilov RF (2003) A microfluidic system for controlling reaction networks in time. Ang Chem Int Ed 42: $768-772$

Song H, Chen D, Ismagilov RF (2006) Reactions in droplets in microfluidic channels. Ang Chem Int Ed 45:7336-7356 
Tan YC, Fisher JS, Lee AI, Cristini V, Lee AP (2004) Design of microfluidic channel geometries for the control of droplet volume, chemical concentration, and sorting. Lab Chip 4:292-298

Teh S, Lin R, Hung L, Lee AP (2008) Droplet microfluidics. Lab Chip 8:198-220

Thorsen T, Roberts WR, Arnold FH, Quake SR (2001) Dynamic pattern formation in a vesicle-generating microfluidic device. Phys Rev Lett 86:4163-4166

van Steijn V, Kreutzer MT, Kleijn CR (2007) $\mu$-PIV study of the formation of segmented flow in microfluidic T-junctions. Chem Eng Sci 62:7505-7514

Voigt E, Albert J, Mayer G, Morgenroth W, Henkel T (2007) All-Glas Bauelemente für die flüssig/flüssig Phasenseparation. In: Proceedings MikroSystemTechnik Kongress, VDE Verlag, Berlin, pp 461-464
Wereley ST, Meinhart CD (2005) Micron-resolution particle image velocimetry. In: Breuer KS (ed) Microscale diagnostic techniques. Springer, New York, pp 55-57

Westerweel J, Scarano F (2005) Universal outlier detection for PIV data. Exp Fluids 39:1096-1100

Zheng B, Tice JD, Ismagilov RF (2004) Formation of droplets of alternating composition in microfluidic channels and applications to indexing of concentrations in droplet-based assays. Anal Chem 76:4977-4982 